\title{
Acoustic Echo Cancellation using Computationally Efficient Adaptive Algorithm Techniques
}

\author{
Mastan Sharif Shaik*, K. Satya Prasad, Rafi Ahamed Shaik, D. Venkata Rao \\ Department of Electrical and Communication Engineering, \\ Lakireddy Balireddy college of Engineering, Mylavaram, 523 230, India \\ *Corresponding author, email: naresh.klu@gmail.com
}

\begin{abstract}
Several sign based LMS adaptive filters, which are computationally free having multiplier free weight update loops, are proposed for acoustic echo cancellation. The adaptive filters essentially minimizes the meansquared error between a primary input, which is the echo, and a reference input, which is either echo that is correlated in some way with the echo in the primary input. The results show that the performance of the signed regressor. LMS algorithm is superior than conventional LMS algorithm, the performance of signed LMS and signsign LMS based realizations are comparable to that of the LMS based filtering techniques in terms of Average Attenuation and computational complexity.
\end{abstract}

Keywords: LMS, Acoustic echo cancellation, Mean-Square Error

Copyright $\odot 2016$ APTIKOM - All rights reserved.

\section{Introduction}

Echo is the phenomenon in which the waveforms are repeated due to reflection from the points, where the characteristics of the medium through which the wave propagate changes. Acoustic echoes are due to a feedback path set up between the speaker and microphone in a mobile phone, hand-free phone, teleconference, Voice over IP (VoIP), Voice over Packet (VoP) and hearing aid systems. Acoustic echo is a common problem with audio conferencing system. It originates in the local audio loop-back that occurs when our microphone picks up audio signals from our speaker, and sends it back to the other participant with our voice. The other participant hears this echo of his or her own voice as he or she speaks.

Acoustic echo can be caused when very sensitive microphones are used, speaker volume is turned up very high, or the microphone and speaker are very close to one another. The perceptual effects of an echo depend on the time delay between the incident and reflected waves, the strength of the reflected waves and the number of paths through which the wave is reflected. Due Acoustic echoes communication may be distorted. The acoustic feedback echoes are desirable in music but undesirable for speech as they are annoying and hamper the quality of speech. Figure 1 shows teleconference system with Echo paths of room.

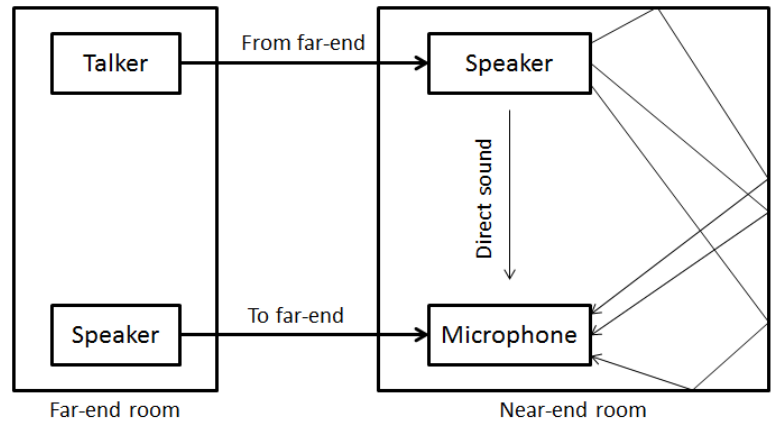

Figure 1. Teleconference system with Echo paths of room 
In this paper we proposed three sign based LMS algorithms in addition to the conventional LMS algorithm for Acoustic echo cancellation. Due to these sign based algorithms the computational complexity (number of multiplications and additions) is reduced and they converges very fast (i.e takes less time to obtain minimum mean square error).

\section{Literature Survey}

The development of Echo cancellation began in 1967 [1], and continues today as an active field of research due to the digital wireless communication systems. The main cause of the problem was longer propagation delays. Different approaches have been given in the literature for acoustic echo cancellation [2]-[8]. Currently, adaptive filtering has become one of the most effective and popular approach for the acoustic echo cancellation due to its simplicity and computational complexity. The fundamental principles of LMS adaptive filtering in stationary and nonstationary environment were described by widrow et al. [9]. Based on the LMS algorithm several papers have been presented in the area of Acoustic echo cancellation [10]-[13]. The reference inputs to the LMS algorithm are deterministic functions and are defined by a periodically extended, truncated set of orthogonal basis functions. In such a case, the LMS algorithm operates on an instantaneous basis such that the weight vector is updated for every new sample within the occurrence based on an instantaneous gradient estimate. In a recent study, however, a steady state convergence analysis for the LMS algorithm with deterministic reference inputs showed that the steady state weight vector is biased, so the adaptive estimate does not approach the Wiener solution. To handle this problem, another procedure was considered for estimating the coefficients of the linear expansion, namely, Block LMS (BLMS) algorithm [14], in which the coefficient vector is updated only once for every occurrence based on a block gradient estimation. The major advantage of the block or the transform domain LMS algorithm is that the input signals are approximately uncorrelated. Complexity reduction of the Acoustic echo cancellation system, particularly in applications such as wireless digital communication systems has remained a topic of intense research from last two decades. This is because of the fact that with the increase in data transmission rate, the channel IR(information rate) length increases and thus the order of the filter increase. The resulting increase in complexity makes the real time operation of the Acoustic echo cancellation system difficult, Especially in view of simultaneous shortening of the signal period, which means that lesser and lesser time will be available to carry out the computations while the volume of computations goes on increasing. The complexity can go further if one employs fast converging equalizers such as those belonging to the RLS family. Thus far, to the best of our knowledge, no effort has been made to reduce the computational complexity of the adaptive algorithm without affecting the signal quality. In order to achieve this, we considered the sign based algorithms. These algorithms enjoy less computational complexity because of the sign present in the algorithm. In [15], MZU Rahman et al. these techniques are applied for cancelling artifacts in ECG signals. In the literature, there exist three versions of the signed LMS algorithm, namely, the signed Regressor algorithm, the sign algorithm and the sign-sign algorithm. All these algorithms require only half as many multiplications as in the LMS algorithm, thus making them attractive from practical implementation point of view [16]-[17]. This paper is organized as follows: in section 2 several computationally efficient adaptive algorithms are described. Section 3 presents Matlab simulations results, the computationally complexity issues are drawn in Section 4 and the conclusions are given in Section 5.

\section{Computationally Efficient Adaptive Filter for Acoustic Echo Cancellation (Proposed Techniques)}

The current digital communication systems such as Public Switched Telephone Network (PSTN), Voice over IP (VoIP), Voice over Pocket (VoP) and mobile phone networks; the need of Acoustic echo cancellation(AEC) is very important and necessary because to bring the better voice quality of the service and to obtain the main purpose of the communication service providers. Figure 2 shows an Acoustic echo canceller using an adaptive filter. The basic functions of the acoustic echo canceller using adaptive filter are estimate the characteristics of echo path, create a replica of the echo and subtract the echo to obtain the desired signal.

The acoustic echo canceller's aim is to detect and remove echo, thereby enhancing the voice quality of the near-end speech $v(n)$. The echo is obtained by filtering the far-end speech $x(n)$ by the echo path vector $y(n)$ of length $L$. The microphone signal $d(n)$ is the echo $y(n)$ plus the near-end speech $v(n)$ and background noise $\mathrm{c}(\mathrm{n})$ which is expressed as

$$
d(n)=y(n)+v(n)+p(n)=h T(n) x(n)+v(n)+c(n)
$$

APTIKOM J. CSIT Vol. 1, No. 2, 2016 : 57-62 
where the superscript ()T denotes the transpose.

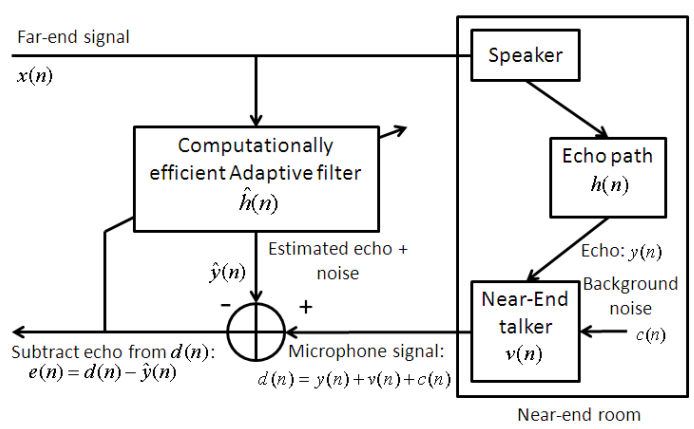

Figure 2. Acoustic echo cancellation using adaptive filter

The error signal $e(n)$ is obtained by subtracting the estimated echo plus noise $\hat{y}$ (n) from the desired signal d(n).

$$
\mathrm{e}(\mathrm{n})=\mathrm{d}(\mathrm{n})-\hat{y}(\mathrm{n})=\mathrm{y}(\mathrm{n})+\mathrm{v}(\mathrm{n})+\mathrm{c}(\mathrm{n})-\hat{y}(\mathrm{n})
$$

Since its development, LMS algorithm is the widely used adaptive algorithm for an acoustic echo cancellation due to its simplicity, robustness and low computational complexity. This section describes the computationally efficient algorithms starting from the fundamental LMS algorithm.

\subsection{The Least Mean Square (LMS) Algorithm}

The LMS algorithm is a stochastic implementation of the steepest descent algorithm. It changes its filter tap weights to converge to the optimal Wiener solution. It simply replaces the cost function $\xi(n)=E[e 2(n)]$ by its instantaneous coarse estimate.

where $\mathrm{E}[$.$] is the expectation operator.$

The error estimation $\mathrm{e}(\mathrm{n})$ is

$$
e(n)=d(n)-y(n)=d(n)-h T(n) x(n)
$$

The Coefficient update recursion is

$$
h(n+1)=h(n)+\mu x(n) e(n)
$$

where; $h(n)=[h 0(n), h 1(n), \ldots, h L-1(n)] T$ is the L-th order adaptive filter.

$\mathrm{x}(\mathrm{n})=[\mathrm{x} 0(\mathrm{n}), \mathrm{x} 1(\mathrm{n}), \ldots, \mathrm{xL}-1(\mathrm{n})] \mathrm{T}$ is the input vector. and $\mu$ is an appropriate step size parameter. For the convergence of the algorithm $\mu$ is chosen as $0<\mu<(2 / \operatorname{tr} R)$.

where $\mathrm{R}$ is the autocorrelation function. $\mathrm{R}=\mathrm{E}[\mathrm{x}(\mathrm{n}) \mathrm{xT}(\mathrm{n})]$ and $\operatorname{tr} \mathrm{R}$ is the trace of the vector matrix $\mathrm{R}$.

\subsection{The Signed-Regressor Lms (SRLMS) Algorithm}

The Signed-Regressor algorithm is obtained from the conventional LMS recursion by replacing the tap-input vector $\mathrm{x}(\mathrm{n})$ with the vector $\operatorname{sign}(\mathrm{x}(\mathrm{n}))$. The SRLMS algorithm is very stable for Acoustic Echo cancellation and also for Gaussian inputs. The adaptive filter coefficients are updated by the SignedRegressor LMS algorithm recursion as

$$
h(n+1)=h(n)+\mu \operatorname{sign}(x(n)) e(n)
$$

where $\operatorname{sign}($.$) is the Signum function$ 
By the replacement of $x(n)$ by its sign, implementation of this recursion may be cheaper than the conventional LMS recursion, especially in high speed real-time applications such as Hearing-Aid systems, Mobile communication these types of recursions may be needed.

\subsection{The Signed Lms (SLMS) Algorithm}

This algorithm is obtained from conventional LMS recursion by replacing the error vector e(n) with the vector $\operatorname{sign}(\mathrm{e}(\mathrm{n}))$. Here Sign is applied to the error signal. So the Signed LMS algorithm recursion is

$$
h(n+1)=h(n)+\mu \quad x(n) \operatorname{sign}(e(n))
$$

\subsection{The Signed Lms (SLMS) Algorithm}

This algorithm can be obtained by combining Signed-Regressor LMS and Signed LMS recursions. By using only signs of input and error signal, the computational complexity is greatly reduced. Hence the SSLMS algorithm recursion is

$$
h(n+1)=h(n)+\mu \operatorname{sign}(x(n)) \operatorname{sign}(e(n))
$$

\subsection{Figure of Merits}

The aim of the echo canceller is to perfectly remove any emanating signal for retrieving the original speech signal. The quality of the proposed algorithms is measured in terms of Mean-Square Error (MSE), Average Attenuation (AV) and Echo Return Loss Enhancement (ERLE).

$$
\begin{array}{ll}
\text { MSE in } \mathrm{dB} & =10 \log 10(\|e(n)\|) 2 \\
\mathrm{AV} \text { in } \mathrm{dB} & =-10 \log 10\{\mathrm{x}(\mathrm{n}) / \mathrm{e}(\mathrm{n})\} 2 \\
\text { ERLE in } \mathrm{dB} & =10 \log 10\{\mathrm{E}[\hat{y} 2(\mathrm{n})] / \mathrm{E}[\hat{e} 2(\mathrm{n})]\}
\end{array}
$$

Where $\hat{e}(\mathrm{n})=\mathrm{x}(\mathrm{n})-\hat{y}(\mathrm{n})$

\section{Matlab Simulations}

The adaptive filtering algorithms presented in section 4 were simulated using Matlab. The echo was created by considering echo delay as $64 \mathrm{~ms}$. The adaptive filter is a 1025 th order FIR filter. The step size was set to 0.01 for all the algorithms. Figure 3 shows the input signal, input signal plus echo, the output signal and the learning curve (MSE behavior) of the LMS algorithm. The Learning curve of the LMS algorithm shows that as the algorithm progresses the average value of the cost function decreases. Figure 4 shows the results of the SRLMS adaptive echo cancellation simulations The adaptive echo cancellation simulation results of the SLMS algorithm are depicted Figure 5. Figure 6 shows the simulation results for the SSLMS algorithm for adaptive echo cancellation. The Learning curve of the SSLMS algorithm shows that the convergence rate is poor compare to the SRLMS. A summary of the performance of the simulated adaptive algorithms is presented in Table 1. 

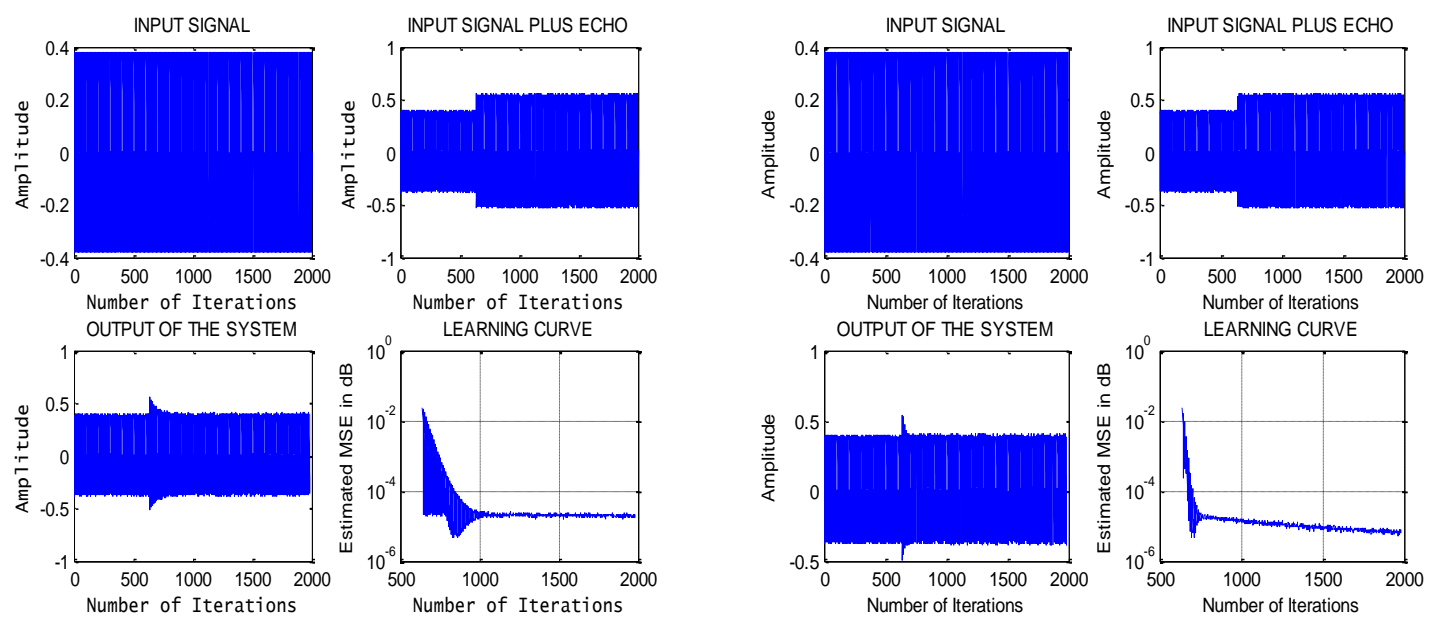

Figure 3. Input, output and convergence characteristics of LMS

Figure 4. Input, output and convergence characteristics of SRLMS
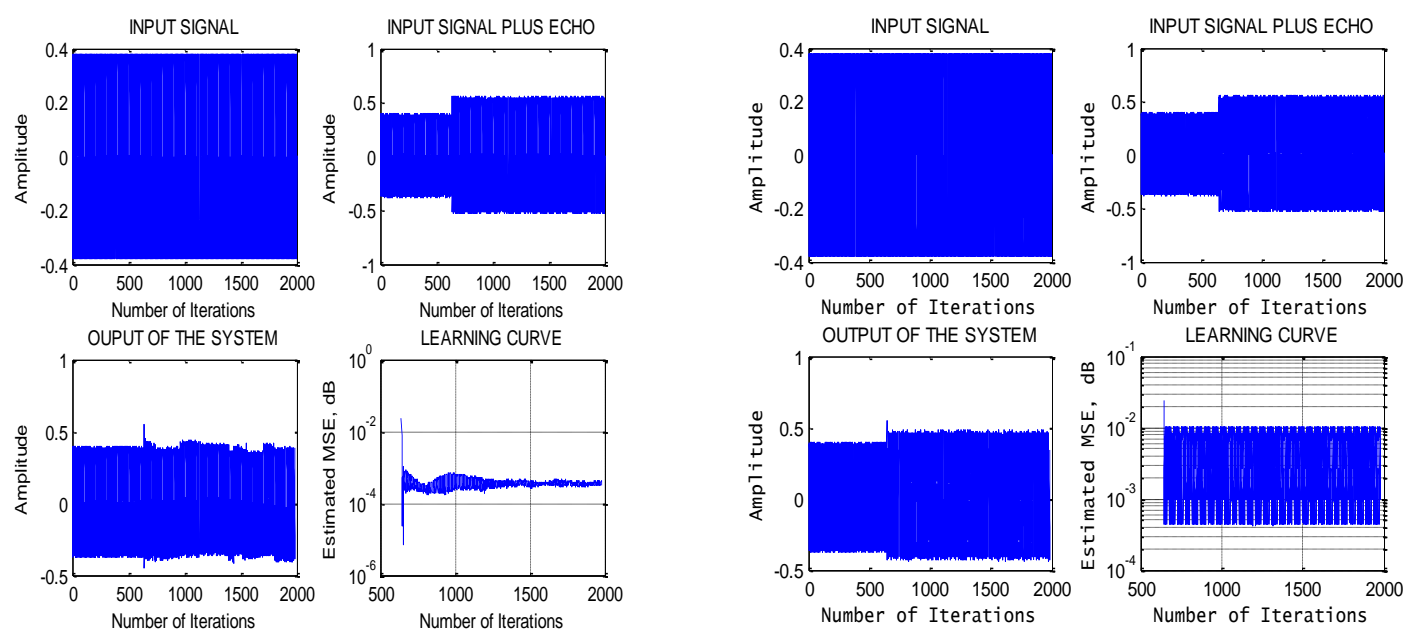

Figure 5. Input, output and convergence characteristics of SLMS

Figure 6. Input, output and convergence characteristics of SSLMS

Table 1. Summary of adaptive algorithms performance

\begin{tabular}{cccc}
\hline Algorithm & MSE in dB & AV in dB & ERLE in dB \\
\hline LMS & -35.665 & -68.947 & 18.501 \\
SRLMS & -39.529 & -40.718 & 18.093 \\
SLMS & -34.558 & -21.788 & 27.554 \\
SSLMS & -25.104 & -23.076 & 26.506 \\
\hline
\end{tabular}

\subsection{Computational Complexity Issues}

The computational complexity figures to compute all the three versions of Sign based LMS algorithms as proposed above are summarized in Table 2. The sign based algorithms offer significant reduction in the number of operations required for conventional LMS algorithm. Further as these algorithms are largely free from multiplication operation, so significant reduction in transit time of the system obtained, which is the main requirement for the acoustic echo cancellation. For LMS algorithm L+1 multiplication and $\mathrm{L}+1$ addition are required to compute the weight update equation 4 . In case of SRLMS algorithm only one multiplication is required to compute $\mu \mathrm{e}(\mathrm{n})$. Whereas other two SLMS and 
SSLMS algorithms does not require multiplication if we choose $\mu$ value a power of 2 . In these cases multiplication becomes shift operation which is less complex in practical realizations.

Table 2.Computational complexity comparison Table

\begin{tabular}{cccc}
\hline Algorithm & Multiplications & Additions & Shifts \\
\hline LMS & L+1 & L+1 & NIL \\
SRLMS & 1 & L+1 & NIL \\
SLMS & NIL & L+1 & NIL \\
SSLMS & NIL & L+1 & NIL \\
\hline
\end{tabular}

\section{Conclusion}

In this paper the problem of acoustic echo cancellation using classical LMS, SRLMS, SLMS and SSLMS are presented and simulated to choose the best implementation. Due to its simplicity the LMS algorithm is the most popular but it suffers with computational complexity, slow and data-dependent convergence behavior. The SRLMS has less computational complexity and exhibits good convergence characteristics than LMS algorithm. The SLMS and SSLMS has very less computational complexity but they exhibits poor convergence. Taking into consideration both number of multiplications (Hardware Complexity) and Mean-Square Error behavior (convergence speed) the SRLMS has been largely used in real-time applications.

\section{References}

[1] M.M.Sondhi, An adaptive echo canceller, Bell System technical journal, XLVI (3), 497-510, March (1967).

[2] Ioana Homana, Marina Dona Topa, Botand Sandor Kirei-Echo Cancellation Using Adaptive Algorithms- IEEE 9781-1-4244-50330309-2009

[3] Tiange Shao, Rosa Zheng and Jacob Benesty, A variable step-size normalized sign algorithm for Acoustic Echo Cancellation, IEEE, 978-1-4244-4296, 2010.

[4] Vojko Pohor and Alberto Cairini, A variable step-size control of adaption for acoustic echo cancellation filters, Wiley On Line Library, European Transactions on Telecommunications,vol.13,issue.2,sep 2008.

[5] Peter Heitkamper, An adaption control for Acoustic Echo Cancellers, IEEE, Vol.4, No.6, June 1997.

[6] Cristina Gabriela Sarachin et al, Echo cancellation using the LMS algorithm,U.P.B Sci Bull series C, Vol. 71,Issue.4, 2009.

[7] John F.Doherty and Rajiv Porayath, A Robust Echo canceller for Acoustic Environments, IEEE, Vol. 44, No.5,May 1997.

[8] Peter Eneroth, Jacob Benesty et al, State of the art of Stereophonic Acoustic Echo Cancellation, Research Gate.

[9] Widrow et al, Stationary and non stationary characteristics of the LMS adaptive filter, IEEE, Vol.64, No.8, August (1976).

[10] Christiane Antweiler et al, Approximation of optimum step-size control for Acoustic Echo cancellation, IEEE, 0-8186-7919-0/97, 1997.

[11] Kousik Ghose, V. Umapathi Reddy, A double talk detector for acoustic echo cancellation applications, ELSEVIER, Signal Processing 80(2000) 1459-1467.

[12] Farhang Boroujeny- Fast LMS/Newton Algorithms based Autoregressive modeling and their applications to Acoustic Echo cancellation - IEEE Transactions and Signal Processing, Vol 45, No.8, August 1997.

[13] A.Zerguine, C.F.N.Cowan and M.Bettayeb- Adaptive Echo Cancellation Using Least Mean Mixed Norm Algorithm-IEEE 1053-587X/97- 1997.

[14] M.Chakraborty, Rafi Ahamed Shaik and Moon Ho Lee, A Block Floating Point based Realization of the Block LMS algorithm, IEEE, Vol.53, No.9, September, 2006.

[15] MZU Rahaman, Rafi Ahamed Shaik and D.V.Rama Koti Reddy, Efficient sign based normalized adaptive filtering techniques for cancellation of artifacts in ECG signals: Application to wireless biotelemetry, ELSEVIER, Signal Processing 91(2011) 225-239.

[16] B.Farhang Boroujeny- Adaptive Filters Theory and Applications, John Wiley \& Sons- 2000.

[17] Haykin Simon- Adaptive Filter Theory, Prentice Hall International Inc-1986. 